\title{
GESTIÓN INTEGRAL DE RESIDUOS SÓLIDOS EN LA EMPRESA CYRGO SAS
}

\author{
INTEGRAL MANAGEMENT OF SOLID WASTE \\ IN THE COMPANY CYRGO SAS \\ GESTÃO INTEGRADA DE RESÍDUOS SÓLIDOS \\ NA EMPRESA CYRGO SAS
}

\author{
Por: LEITON RODRIGUEZ _ Natalia Vanessa, REVELO MAYA_Wilson Guillermo
}

Especialista en gerencia de proyectos, Corporación Universitaria Minuto de Dios, Coinvestigadora Universidad de Nariño. Email: natalialeiton@hotmail.com, Colombia.

Magister en Administración, Universidad del Valle, Director Departamento de Administración de Empresas y Finanzas, Universidad de Nariño. Email wilrev@gmail.com, Colombia.

DOI: http://dx.doi.org/10.22267/rtend.171802.79

\section{RESUMEN}

El presente trabajo de investigación, consiste en la elaboración de un Plan de Gestión Integral para el manejo de residuos sólidos en la empresa Cyrgo SAS, cuyo objetivo general radica en la formulación de dicho Plan. La metodología diseñada es Investigación de Tipo Descriptivo Exploratorio con enfoque mixto; cualitativo - cuantitativo y se construye con un diagnóstico completo realizado en cuanto al manejo actual de residuos sólidos en la empresa, con el fin de establecer las medidas a implementar y estructurar un Plan de seguimiento a las actividades propuestas. Los resultados obtenidos a través del trabajo de campo e interacción con las diferentes personas y áreas objeto de estudio, permiten obtener un documento con el conjunto de medidas a implementar para que la empresa lo adopte y de alguna u otra forma pueda disminuir los impactos ambientales negativos que se puedan generar por un inadecuado manejo de residuos sólidos.

Palabras clave: gestión, residuos, normatividad, plan, clasificación.

JEL: M11, Q51, Q52, Q53. 


\begin{abstract}
This research work is the development of an Integrated Management Plan for solid waste management in the company Cyrgo SAS, whose overall objective is to formulate the plan. The research methodology is designed descriptive exploratory type with mixed approach; qualitative - quantitative and built with a complete diagnosis made on the current solid waste management in the company, in order to establish the measures to implement and structure a monitoring plan proposed activities. The results obtained through fieldwork and interaction with different people and areas under study; allow obtaining a document with a set of measures to implement for the company to adopt it and some way to reduce the negative environmental impacts that may arise from improper handling of solid waste.
\end{abstract}

Keywords: management, waste, regulation, plan, classification.

JEL: M11, Q51, Q52, Q53.

\title{
RESUMO
}

Este trabalho de investigação é a desenvolver de um plano de gestão abrangente para gestão de resíduos sólidos na companhia Cyrgo SAS, cujo objetivo geral encontra-se na formulação do plano. A metodologia projetado é pesquisa descritiva exploratório com abordagem mista descritiva; Qualitativo - quantitativa e constrói um diagnóstico completo feito sobre a atual gestão de resíduos sólidos na empresa, a fim de estabelecer as medidas a implementar e estruturar um plano de monitoramento propostos atividades. Os resultados obtidos através do trabalho de campo e interação com pessoas diferentes e áreas em estudo, permitem obter um documento com o conjunto de medidas a serem implementadas pela empresa para adotá-la e de alguma outra forma pode reduzir os impactos ambientais negativos que podem surgir a partir de manuseio inadequado de resíduos sólidos.

Palavras-chave: gestão, resíduo, regulação, plano, classificação.

JEL: M11, Q51, Q52, Q53.

\section{INTRODUCCIÓN}

Los residuos sólidos son el subproducto de la actividad del hombre y se han producido desde los albores de la humanidad. Cada día aumentan en cantidad y variedad como consecuencia del incremento de la población humana y del desarrollo tecnológico e industrial. Su disposición final incorrecta ha ocasionado grandes problemas al ambiente, contaminando agua, aire y suelo.

La problemática ambiental generada por el incremento de los residuos sólidos se debe, en parte, a la falta de educación y responsabilidad ambiental para separarlos en la fuente y poder aprovecharlos nuevamente como materia prima para la fabricación de nuevos productos. El Manejo Integral de los Residuos Sólidos (MIRS) contribuye al ahorro sostenible de los recursos naturales, para resolver la problemática actual se formula la siguiente pregunta ¿Qué factores y situaciones estratégicas se deben considerar en la 
formulación de un Plan de Gestión Integral de Residuos Sólidos en la Empresa Cyrgo S.A.S, ciudad de Pasto departamento de Nariño?

En muchos establecimientos generadores de Residuos Sólidos se han elaborado e implementado Planes de Gestión de Residuos Sólidos, para brindar las respectivas medidas de manejo que permitan llevar a cabo procedimientos para la implementación de acciones en cuanto al manejo integral de los residuos desde su generación hasta su disposición final, lo que permitirá de alguna forma estandarizar los procesos, bajo una política de calidad partiendo de la misión y visión para que desde la gerencia se establezcan las directrices a seguir que serán de obligatorio cumplimiento.

Dentro de la investigación se propone el desarrollo de los siguientes objetivos: realizar un diagnóstico de la situación actual de la organización en el manejo de residuos sólidos, establecer las medidas para el manejo integral de los residuos sólidos generados en la empresa, estructurar un Plan de seguimiento a las actividades planteadas y diseñar un sistema de indicadores de Gestión Integral para el Manejo de Residuos.

El documento Plan de Gestión Integral de Residuos Sólidos en el Establecimiento Cyrgo SAS, se elabora con el fin de que sirva como guía de consulta, texto de capacitación, instructivo y soporte de estandarización de cada una de las actividades desde la generación, segregación, recolección, transporte, almacenamiento temporal y disposición final de residuos peligrosos, de igual forma, contiene actividades las cuales pretenden crear un clima adecuado que promueva y facilite la aplicación de la normatividad vigente, acompañado del estímulo de la responsabilidad social y ambiental, y la invitación a la aplicación de tecnologías más limpias que acompañen la disposición final controlada en el marco de mejora continua.

\section{REVISIÓN DE LA LITERATURA}

2.1. Residuos Sólidos. De acuerdo al Decreto 1713 de 2002 un residuo sólido es cualquier objeto, material, sustancia o elemento sólido resultante del consumo o uso de un bien en actividades domésticas, industriales, comerciales, institucionales, de servicios, que el generador abandona, rechaza o entrega y que es susceptible de aprovechamiento o transformación en un nuevo bien, con valor económico o de disposición final.

2.2. Clasificación de Residuos. De acuerdo al Decreto 4741 de 2005, los residuos son clasificados en Peligrosos y No Peligrosos.

2.3. Residuos peligrosos. Son aquellos desechos que por sus características corrosivas, reactivas, explosivas, tóxicas, inflamables, infecciosas o radiactivas pueden causar riesgo o daño a la salud humana y al ambiente. Así mismo, se considera residuo o desecho peligroso los envases, empaques y embalajes que hayan estado en contacto con ello. Para hacer referencia a este tipo de residuos comúnmente se utiliza el acrónimo RESPEL. Un residuo es peligroso si presenta al menos una de las siguientes características (CRETIBER), bajo las condiciones señaladas en el Anexo III, del Decreto 4741 de 2005. 
Corrosividad: esta característica identifica a aquellos residuos que pueden provocar un riesgo a la salud humana o al ambiente debido a su habilidad para: Movilizar, Corroer y/o Destruir.

Reactividad: los residuos reactivos son aquellos normalmente inestables y que pueden llegar a reaccionar violentamente sin explosión; pueden formar una mezcla explosiva con el agua, generar gases tóxicos, vapores y humos; pueden contener cianuro o sulfuro y generar gases tóxicos.

Explosividad: un residuo posee esta característica cuando es capaz de producir una reacción o descomposición detonante o explosiva solo o en presencia de una fuente de energía o si es calentado bajo confinamiento.

Toxicidad: un residuo es tóxico si tiene el potencial de causar la muerte, lesiones graves, efectos perjudiciales para la salud del ser humano, si se ingiere, inhala o entra en contacto con la piel.

Inflamabilidad: un residuo o desecho es inflamable cuando en presencia de una fuente de ignición, puede arder bajo ciertas condiciones de presión y temperatura. Las muestras de residuos pueden tener líquidos inflamables, sólidos inflamables y gases inflamables.

Riesgo biológico: esta característica identifica a aquellos residuos capaces de provocar una enfermedad infecciosa. Un residuo se considerara infeccioso si contiene microbios patógenos con suficiente virulencia y en tal cantidad, que la exposición al residuo por parte de un huésped sensible puede derivar en una enfermedad infecciosa.

Radiactividad: un residuo presenta esta característica si una muestra representativa del mismo emite espontáneamente radiaciones a un nivel mayor que el de base. Radiación significa la emisión de alguno o algunos de estos elementos: neutrones alfa, beta, gama, o rayos X; y electrones de alta energía, protones u otras partículas atómicas; exceptuando ondas de sonido o de radio y de luz visible infrarroja o ultravioleta.

\section{ROTULADO Y ETIQUETADO DE LOS RESIDUOS O DESECHOS PELIGROSOS}

Los residuos peligrosos deben estar correctamente etiquetados, identificando claramente su contenido y acompañados de un pictograma que indica su característica de peligrosidad, es decir, si se trata de un residuo corrosivo, reactivo, explosivo, tóxico, inflamable o de riesgo biológico (Martínez, 2005), tal como lo establece la Norma Técnica Colombiana NTC 1692 (Ministerio de Ambiente Vivienda y Desarrollo Territorial. República de Colombia, 2007) y teniendo en cuenta los sistemas de señalización homologados en el País. (Betancur, 2011).

III.1. Residuos no peligrosos: son aquellos producidos por el generador en cualquier lugar y en desarrollo de su actividad, que no presentan riesgo para la salud humana o para el medio ambiente, Decreto 4741 de 2005. 
III.2. Reciclables: Son aquellos que no se descomponen fácilmente y pueden volver a ser utilizados en procesos productivos como materia prima. Entre estos residuos se encuentran:

- Chatarra

- Vidrio

- Telas

- Partes y equipos obsoletos o en desuso

- Papeles y plásticos

III.3. Biodegradables: Son aquellos restos químicos o naturales que se descomponen fácilmente en el ambiente. En estos restos se encuentran:

- Vegetales

- Residuos alimenticios no infectados

- Papel higiénico

- Jabones y detergentes biodegradables

- Madera

- Otros residuos que puedan ser transformados fácilmente en materia orgánica

III.4. Ordinarios o comunes: Son aquellos generados en el desempeño normal de las actividades. Estos residuos se generan en oficinas, pasillos, áreas comunes, cafeterías, salas de espera, auditorios y en general en todos los sitios del establecimiento del generador.

III.5. Inertes: Son aquellos que no permiten su descomposición, ni su transformación en materia prima y su degradación natural requiere grandes períodos de tiempo. Entre estos se encuentran: el icopor, algunos tipos de papel como el papel carbón y algunos plásticos.

\section{PLAN DE GESTIÓN INTEGRAL DE RESIDUOS SÓLIDOS, PGIRS}

De acuerdo a la Resolución 1045 de 2003 es un conjunto ordenado de objetivos, metas, programas, proyectos y actividades, definidos por el ente territorial para la prestación del servicio de aseo, basado en la política de Gestión Integral de Residuos Sólidos, el cual se obliga a ejecutar durante un período determinado, basándose en un diagnóstico inicial, en su proyección hacia el futuro y en un Plan Financiero Viable que permita garantizar el mejoramiento continuo de la prestación del servicio de aseo, evaluado a través de la medición de resultados.

IV.1 Separación en la fuente. Para hacer la separación desde la fuente de los residuos sólidos se requiere educar al generador y más allá de decirle que con eso salvará al planeta es poderle generar un nuevo hábito (un nuevo aprendizaje) para que logre mantenerse en la labor de separar sus residuos. Debemos separarlos de tal forma que no se contaminen con otros residuos, cosa que usualmente pasa cuando se mezcla el papel con los envases que suelen contener líquidos que terminan afectando la calidad del papel. 
En Colombia se tiene esta guía para la identificación de las canecas por código de colores.

Clasificación dada por la Guía Técnica Colombiana 24 de 2009 (Figura 1).

Figura No. 1. Código de colores para la Clasificación de Residuos

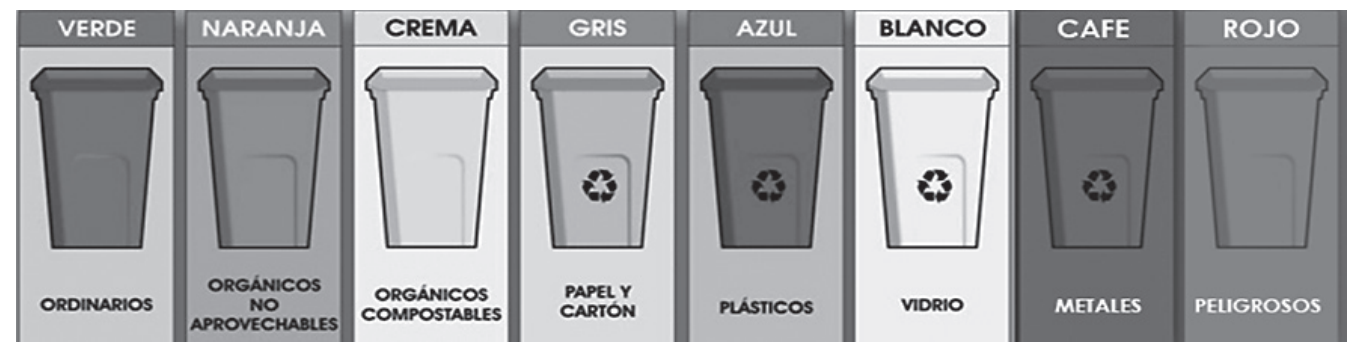

Fuente: guía técnica Colombiana GTC 24 (2009)

IV.2 Almacenamiento temporal. De acuerdo al Decreto 2676 de 2000, es la acción del generador consistente en depositar segregada y temporalmente sus residuos, por un tiempo determinado con carácter previo a su aprovechamiento y/o valorización, tratamiento y/o disposición final.

El almacenamiento temporal de los residuos peligrosos debe cumplir con ciertas características técnicas conforme a lo establecido en el Decreto 4741 de 2005, debe cumplir con las condiciones necesarias para esta actividad, esto disminuirá los impactos ambientales y contribuirá a garantizar la seguridad de las personas.

IV.3 Aprovechamiento y/o Valorización. De acuerdo al Decreto 4741 de 2005 es el proceso de recuperar el valor remanente o el poder calorífico de los materiales que componen los aceites lubricantes usados, por medio de la recuperación, el reciclado o la regeneración.

IV.4 Disposición Final. De acuerdo al Decreto 4741 de 2005 es el proceso de aislar y confinar los aceites lubricantes usados, en especial los no aprovechables, en lugares especialmente seleccionados, diseñados y debidamente autorizados, para evitar la contaminación y los daños o riesgos a la salud humana y al ambiente.

A nivel internacional se encontraron los siguientes estudios:

- En la Universidad Nacional Autónoma de México, se elaboró el Estudio "Plan de Manejo Integral de Residuos Sólidos para Hipermercados” por el autor Geovanelli López Morfin, para optar el título de Ingeniero Industrial, ciudad Universitaria, México D.F, año 2014, cuyo objetivo general era desarrollar la metodología para elaborar un plan de manejo de residuos sólidos para hipermercados y aplicarla, como caso de estudio, en una tienda Wal-Mart de la Ciudad de México. 
- En la Escuela Superior Politécnica de Chimborazo, Facultad de Ciencias, año 2011, se elaboró la tesis para obtener el título de Ingeniero en Biotecnología Ambiental "Propuesta para el Manejo Integral de residuos sólidos generados en el Mall de los AndesAmbato", por la autora Norma Yolanda Gaibor Vaca, Riobamba Ecuador año 2013. La propuesta contempla como objetivo general: realizar una propuesta con sustento técnico y ambiental para el manejo integral de los residuos sólidos generados en el Mall de los Andes de la ciudad de Ambato.

A nivel Nacional se encontraron las siguientes referencias en manejo de residuos:

- Se encontró un estudio "Plan de Gestión Integral de Residuos Sólidos para la Vicepresidencia de Transporte de Ecopetrol S.A", elaborado en la Universidad Industrial en Santander, para obtener el título como Especialización en ingeniería a Ambiental, por los autores Floralba Ferrer Jaramillo y Paola Alexandra Medina Gómez, año 2010. Dicho estudio contemplo como objetivo general: Contribuir a la prevención del impacto ambiental mediante la disminución de residuos sólidos y la realización de prácticas sistémicas de selección, segregación, transporte, almacenamiento, tratamiento y disposición de dichos residuos, asegurando los recursos que permitan la gestión oportuna en cada una de las etapas, dando cumplimiento a la normatividad vigente.

- Por otro lado se encontró otro estudio "Formulación del Plan de Manejo Integral de Residuos Sólidos del Centro Comercial San Pedro Plaza de la Ciudad de Neiva - Huila” en la Pontificia Universidad Javeriana de la ciudad de Bogotá DC, año 2010, dicho estudio establece como objetivo general crear un plan de manejo integral de residuos sólidos para el centro comercial San Pedro Plaza en la ciudad de Neiva, con la intención de empezar a generar un cambio en la cultura del manejo de los residuos sólidos en la urbe.

A nivel Regional no se encontraron como tal estudios en manejo de residuos sólidos, sin embargo se tomó como referencia los siguientes documentos que fueron de bastante utilidad en el proceso de investigación.

- Como referencia para este proyecto en estudios Regionales, se encontró el Plan de Gestión Integral de Residuos Sólidos del municipio de Pasto, año 2007-2022, el cual de acuerdo con lo establecido en el artículo 9 del Decreto 1713 de 2002, el PGIRS se desarrolló a partir de un diagnóstico integral de la situación actual para encontrar las debilidades y fortalezas, de la identificación de posibles escenarios futuros, del diseño y puesta en marcha de programas, proyectos y actividades organizadas en un plan de acción y de la aplicación de un sistema de medición de resultados o programa de seguimiento y monitoreo. Lo anterior con el fin de avanzar hacia condiciones óptimas en un esquema de mejoramiento continuo, con base en la Ley 142 de 1994 y la Política para la Gestión Integral de Residuos establecida por el Gobierno Nacional.

- También se tomó como referencia el Proyecto Programa de Manejo de Residuos Sólidos Regional Nariño, del Instituto Colombiano ICBF, año 2013, el cual a través de un diagnóstico general, ambiental y técnico operativo, permite contar con una línea base desde 
la cual se pueda contemplar estrategias de valorización, aprovechamiento y disposición adecuada de los residuos generados al interior de la institución, minimizando o anulando los impactos ambientales negativos en concordancia con las normas ambientales jurídicas del estado colombiano. (ICBF, 2013).

\section{MÉTODOS Y MATERIALES}

El presente proyecto está enmarcado en una Investigación de Tipo Descriptivo exploratorio con enfoque mixto empírico analítico, paradigma cuantitativo y método deductivo debido a que a través de un Plan de Gestión Integral de residuos sólidos se pretende paso a paso describir todas las actividades a implementar desde la generación hasta la disposición final de residuos, basados en el diagnóstico obtenido de datos que expresan teóricos y numéricos.

La población objeto del presente estudio está conformada por las 31 personas que laboran en Cyrgo SAS que generan residuos en la ejecución de cada una de sus actividades, tanto personal operativo; Servicios Generales, auxiliares de bodega y auxiliares de producción, como administrativo: Gerente Comercial, Jefe de logística, Coordinador de Producción, Coordinador Salud Ocupacional, Compras, Comercial, Auxiliares Contable y de Logística.

Se tomara como muestra para la realización de encuestas los 31 trabajadores de Cyrgo para establecer el diagnóstico de residuos generados.

Para el cumplimiento de los objetivos planteados en la investigación se hace necesario el establecimiento de una metodología coordinada y ordenada, la cual contempla el procedimiento y materiales a utilizar en cada una de las etapas, las cuales se resumen en el presente cuadro (Tabla 1).

Tabla No. 1. Procedimiento y materiales

\begin{tabular}{|c|c|c|}
\hline METODOLOGIA & PROCEDIMIENTO & MATERIALES \\
\hline \multicolumn{3}{|c|}{ Etapa de Diagnóstico } \\
\hline $\begin{array}{l}\text { Identificación de las diferentes áreas que } \\
\text { cuenta la empresa con cada residuo que } \\
\text { genera "Identificación cualitativa". }\end{array}$ & $\begin{array}{l}\text { Visita cada una de las áreas y } \\
\text { descripción de las actividades que se } \\
\text { desarrollan en cada área } \\
\text { Identificación de los residuos sólidos } \\
\text { generados. }\end{array}$ & $\begin{array}{l}\text { Cámara fotográfica, } \\
\text { registros fotográficos. }\end{array}$ \\
\hline $\begin{array}{l}\text { Elaboración de encuestas a los trabajadores } \\
\text { para identificar clases de residuos sólidos } \\
\text { generados y como se generan desde sus } \\
\text { diferentes actividades hasta su disposición; y } \\
\text { los usos que le dan a cada uno de ellos para } \\
\text { detectar los tipos de contaminación ambiental }\end{array}$ & $\begin{array}{l}\text { Elaborar un formato de encuesta } \\
\text { que permita identificar clases de } \\
\text { residuos sólidos generados y como } \\
\text { se generan desde sus diferentes } \\
\text { actividades hasta su disposición } \\
\text { final. }\end{array}$ & $\begin{array}{lr}\text { Formato } & \text { base } \\
\text { encuestas, } & \text { material } \\
\text { bibliográfico. } & \end{array}$ \\
\hline
\end{tabular}


Elaboración de entrevistas a Gerente, para ser aplicado al gerente Comercial de la Empresa Cyrgo SAS.

Identificación del manejo actual de residuos sólidos generados en el establecimiento.

Identificación de cantidades de residuos sólidos generados.

Análisis de involucrados

Análisis de la brecha

Árbol de Problemas
Elaborar un formato de entrevista que permita conocer manejo actual de los residuos generados, tipos de residuos y cantidades aproximadas generadas

Análisis de las entrevistas realizadas a Gerente, y persona de servicios generales.

Recorrido y revisión visual del manejo actual de los residuos sólidos desde su generación hasta su disposición final.

Con base al diagnóstico obtenido de residuos sólidos generados cuantificar la cantidad generada de residuos en el año 2015.

Identificar los actores se benefician directamente con el Proyecto de Manejo de Residuos, a través de la matriz de análisis de involucrados.

Utilizando la Matriz DOFA de Material Bibliográfico Fortalezas y debilidades.

Identificación de los principales problemas actuales, causas y efectos a través del árbol de problemas.

Formato base para entrevista, grabadora, material bibliográfico.

Cámara fotográfica Resultados de las encuestas y entrevistas realizadas

Programa en Excel para análisis estadístico de las encuestas.

Datos históricos.

Matriz análisis de involucrados, material bibliográfico.

Material Bibliográfico

Plan de Gestión Ambiental para el Manejo Integral de residuos sólidos

Definir las medidas de manejo que se implementaran en el Plan de Gestión Ambiental para el Manejo Integral de residuos sólidos teniendo en cuenta el cumplimiento de la normatividad aplicable.

Educación y Formación

Salud Ocupacional e Higiene y Seguridad Industrial.
De acuerdo al diagnóstico realizado se establecen las medidas de manejo a implementar en la gestión de residuos sólidos

Escoger la temática más relevante en el manejo de residuos sólidos y plasmar los diferentes temas que se trataran para una adecuada gestión integral de residuos sólidos.

Plasmar en el documento las medidas a implementar en cuanto a Salud Ocupacional, Seguridad Industrial y Medicina preventiva en el trabajo.
Documentación obtenida en el diagnóstico, material bibliográfico, normatividad en manejo de residuos.

Material bibliográfico

Material bibliográfico 
Definición de un Plan de Contingencia

Definir procedimientos a aplicar en caso de ocurrir situaciones de emergencia.

Seguimiento y Medición

Definición de sistemas de medición, análisis, Tomar las acciones a implementar evaluación y mejora.

como preguntas para colocarlas como criterios de verificación y evaluación en una lista de chequeo, la cual servirá para detectar no conformidades y establecer acciones de mejora continua. Establecimiento de un procedimiento para realización de auditorías internas anuales.
Medidas a implementar en el Plan de Gestión Integral para el manejo de Residuos Sólidos Material bibliográfico Lista de chequeo.

Indicadores de gestión

Diseño de un sistema de indicadores de Definición de los indicadores de Material bibliográfico Gestión Integral para el Manejo de Residuos gestión a evaluar. Sólidos.

Fuente: Elaboración propia de los autores

\section{RESULTADOS}

\section{Diagnóstico de la situación actual de la organización en el manejo de residuos sólidos}

La Empresa CYRGO SAS Pasto cuenta con áreas operativas y administrativas, las cuales son generadoras tanto de residuos sólidos peligrosos y no peligrosos.

De acuerdo al análisis de las encuestas el 53\% de la población encuestada pertenece al área operativa, esto quiere decir que es la población con mayor número de trabajadores por área y los cargos que más predominan son los de auxiliar de bodega con un 33\% del total de la población.

Se puede observar que los porcentajes obtenidos por cada residuo generado son similares, sin embargo se puede evidenciar con un $8 \%$ y un $7 \%$ los residuos que más se generan son el papel, resultado del barrido y papel higiénico y con un 5\% cartón, calamina, chatarra, empaques comestibles, los que menos se generan son papel periódico, baterías productos químicos, sin contaminar. 
Figura No. 2. Clasificación y separación de los residuos sólidos por la población

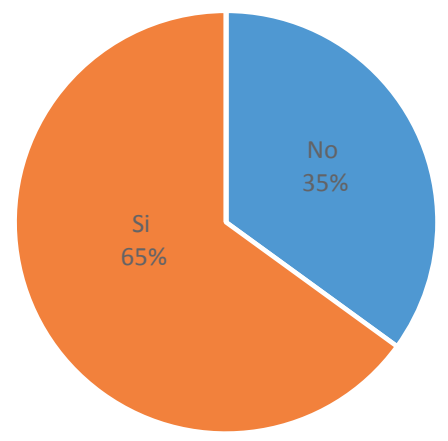

Fuente: Elaboración propia de los autores

Un 65\% en la empresa si clasifica o separa los residuos y un 35\% no, correspondiente al personal administrativo, debido a que no cuenta con los respectivos contenedores para la separación de residuos.

Figura No. 3. Existencia de contenedores para separación de residuos de acuerdo al código de colores (Rojo, verde, azul, gris)

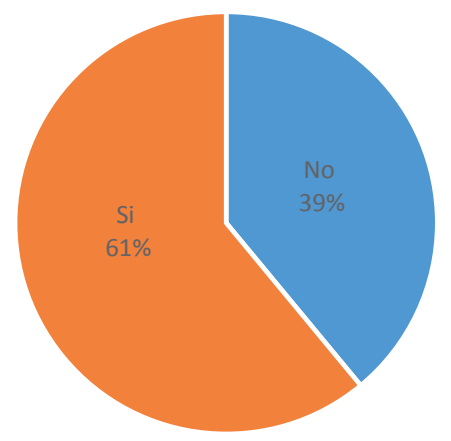

Fuente: Elaboración propia de los autores

El 61\% de la población encuestada manifiesta que si cuentan con contenedores de acuerdo al código de colores, este porcentaje corresponde al personal operativo, mientras que el 39\% manifiesta no tener los contenedores en mención, aquí se puede incluir personal administrativo y ventas.

Conforme a las preguntas que se realizaron a los trabajadores el 91\% afirma contar con bolsas en cada recipiente, sin embargo el $94 \%$ responde que estos no cuentan con tapa. 
Figura No. 4. Lugar de depósito de residuos generados en las áreas de trabajo

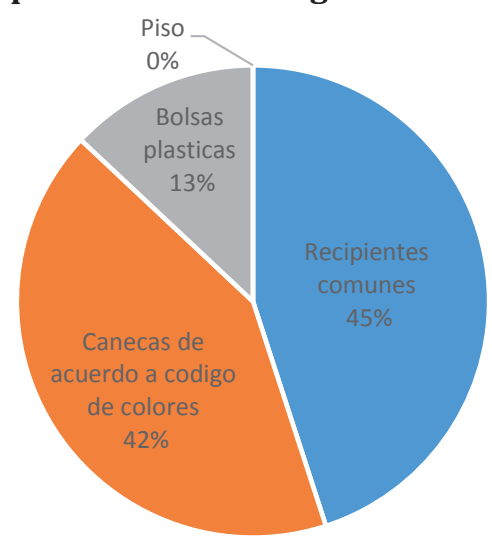

Fuente: Elaboración propia de los autores

El 42\% de la población correspondiente al personal operativo, manifiestan depositar los residuos en canecas de acuerdo al código de colores, el 45\%, entre ventas y administrativo manifiesta depositar los residuos en recipientes comunes y el 13\% los deposita en bolsas.

El 94\% de la población encuestada manifiesta que los recipientes no cuentan con la respectiva tapa y el 6\% que sí. Mostrándose un 94\% como un valor significativo para determinar que los recipientes no cuentan con tapa.

\section{Figura No. 5. Suficiencia de contenedores en la empresa para la separación de residuos}

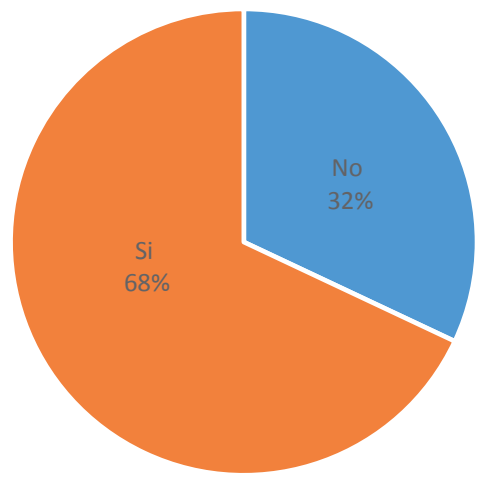

Fuente: Elaboración propia de los autores

Un $68 \%$ de la población encuestada expresa que los recipientes implementados son suficientes, mientras que un 32\% no, de este porcentaje se destacan las áreas administrativas, donde manifiestan la carencia de recipientes para separación de residuos; Un 77\% del personal realmente desconoce la existencia de rutas de recolección. 
Figura No. 6. Almacenamiento temporal de residuos

Fuente: Elaboración propia de los autores

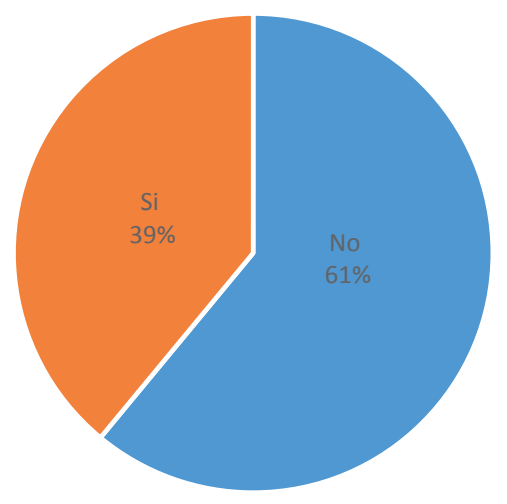

Un 61\% de la población encuestada manifiesta que no cuenta con un sitio de almacenamiento temporal, mientras que un 39\% sí. Sin embargo es otro tema que desconocen, pues la empresa no cuenta con un sitio de almacenamiento temporal de residuos.

\section{Figura No. 7. Capacitación en el manejo de residuos solidos}

Fuente: Elaboración propia de los autores

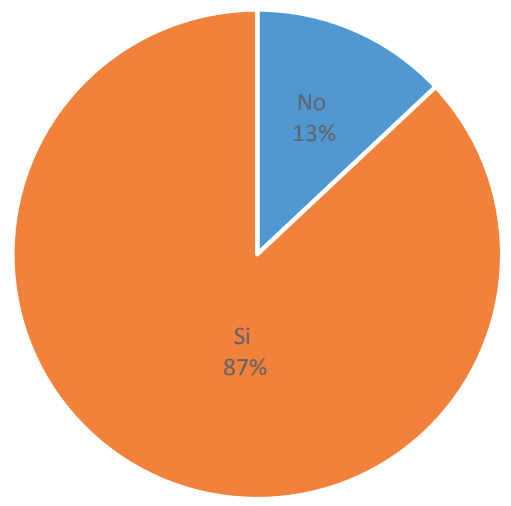

Con un $87 \%$ del personal encuestado, en su gran mayoría manifiestan haber recibido algún tipo de capacitación y un $13 \%$ no ha recibido.

La entrevista realizada a la señora Ángela Arteaga, Gerente Comercial de la Empresa Cyrgo SAS, arroja la carencia de un Plan de Residuos Sólidos, pues hasta el momento no se ha consolidado ningún tipo de documento que permita estandarizar los procesos para un adecuado manejo de residuos.

Hasta el momento las actividades que se han llevado a cabo para el manejo de residuos, es la implementación de recipientes de acuerdo al código de colores (gris, verde, azul y rojo) para separar los residuos en las áreas operativas, se cuenta con 3 puntos ecológicos 
distribuidos al interior de la bodega de la empresa, de la misma forma se están separando los peligrosos de los demás residuos para ser dispuestos con un gestor autorizado.

En las áreas administrativas, aun se cuenta con recipientes ordinarios, lo que se llaman las papeleras y no se hace la separación de residuos, pues como solo hay una por cada puesto de trabajo, todos son dispuestos en el mismo contenedor, Teresa López la encargada de servicios generales, hace la separación manual de estos residuos para tratar de recuperar los reciclables como papel y cartón.

Lastimosamente, no se tienen control de cantidades de residuos sólidos generados, pues no ha existido una directriz ni un formato que permita llevar el control, se pretende que con la implementación del Plan se pueda controlar ese aspecto.

La gerente comercial, reconoce que en las áreas administrativas las cantidades de contenedores implementados no son las suficientes, ni están de acuerdo al código de colores para facilitar la separación de los residuos, así mismo la carencia de un sitio de almacenamiento temporal de residuos, pues se vió la necesidad, pero están a la espera de la aprobación por parte de la gerencia de Bogotá.

Un gran problema detectado es que anteriormente se depositaban los residuos peligrosos como luminarias, baterías, pilas, aceite usado, elementos contaminados con aceite con la basura común por la falta de conocimiento sobre el tema.

La disposición final para los residuos ordinarios e inertes la realizan con la empresa Metropolitana de Aseo EMAS S.A. E.S.P., quien hace la recolección tres veces por semana, en cuanto a los residuos reciclables son entregados a una persona que pertenece a una asociación de recicladores y los peligrosos se tienen almacenados para ser entregados a una gestora autorizada, designada por el área ambiental de la empresa.

Se han realizado capacitaciones generales en temas de manejo de residuos, pero se ve la necesidad de profundizar en temas más específicos para garantizar un adecuado manejo de residuos sólidos.

Se procedió a entrevistar a jefes de bodega y servicios generales, para determinar cantidades aproximadas de residuos generadas mensualmente (Tabla 2).

Tabla No. 2. Cantidades aproximadas de Residuos Sólidos generados

\begin{tabular}{lc}
\multicolumn{1}{c}{ Pregunta } & Conocen \\
Disposición de Residuos Ordinarios e Inertes & 94 \\
Disposición de Residuos Reciclables & 23 \\
Disposición de Residuos Peligrosos & 0 \\
\hline
\end{tabular}

Fuente: Elaboración propia de los autores 
Tabla No. 3. Conocimiento de disposición final de residuos

\begin{tabular}{lcc}
\hline \multicolumn{1}{c}{ Tipo de residuo } & Cantidad generada & $\begin{array}{c}\text { Periodo de } \\
\text { Generación }\end{array}$ \\
Ordinarios e inertes & 1 a 2 canecas de 55 galones & Semanal \\
Reciclables & 3 sacas de papel de archivo y & Semanal \\
Aceite usado & 70 galones & Semestral \\
Luminarias & 10 unidades & Anual \\
Pilas & 50 unidades & Anual \\
Baterías & 1 unidad & Anual \\
Equipos Electrónico en desuso “RAE” & 1 unidad & Anual \\
Tóneres de impresoras o cartuchos. & 4 unidades & 4 meses \\
Frascos de aceite & 3 unidades & Semestral \\
Elementos contaminados con aceite & 3 Kilos & Mes \\
Recipientes productos químicos & 13 Kg & Anual \\
\hline
\end{tabular}

Fuente: Elaboración propia de los autores

Se puede evidenciar además un porcentaje muy alto correspondiente al 94\% conoce donde se realiza la disposición final de residuos ordinarios, mencionando la Empresa EMAS S.A. E.S.P., el 23\% conoce la disposición final de los residuos reciclables y el 0\% conoce la disposición final de residuos peligrosos.

El Manejo actual de residuos desde su generación hasta su disposición final está dado de la siguiente manera:

Segregación de residuos. La segregación de residuos en áreas administrativas se realiza en recipientes comunes, implementados uno por cada área y en las áreas operativas, se encuentran ubicados 3 puntos ecológicos, cada uno con sus cuatro recipientes, de acuerdo al código de colores (verde, gris, azul y rojo). De igual forma se inició con la segregación de residuos como tóners y pilas.

Recolección Interna. La recolección interna de residuos sólidos en áreas administrativas es realizada por la persona encargada del aseo todos los días, una vez al día al finalizar la jornada. Esta recolección es realizada de forma manual y consiste en dirigirse a cada recipiente ubicado en las oficinas, primero toma los residuos reciclables como papel y cartón, los guarda en una bolsa para almacenarlos en el cuarto de aseo y tenerlos listos hasta que los recicladores lo recolecten. Finalmente recolecta la basura común, hace el reembase de cada recipiente a una bolsa para posteriormente disponerlos en los recipientes ubicados en la bodega. 
En el área operativa se disponen los residuos primero en los contenedores pequeños de los dos puntos ecológicos, posterior a esto el trabajador asignado para el aseo, manualmente transporta los residuos en la respectiva bolsa y los dispone en los contenedores de 55 galones ubicados al acceso de la puerta lateral derecha, en este punto la empresa de aseo hace la recolección externa para ser dispuesto finalmente en el relleno sanitario.

Almacenamiento temporal. La empresa no cuenta con un sitio de almacenamiento temporal.

Disposición final. Los residuos ordinarios e inertes son dispuestos con la empresa metropolitana de aseo EMAS S.A. E.S.P., los reciclables son dispuestos con una persona recicladora y los peligrosos actualmente se tienen separados para ser dispuestos con la empresa SERPROINGENIERÍA la cual cuenta con la respectiva Licencia Ambiental.

Los principales actores en el manejo de residuos son el Personal Administrativo, Operativo y de Servicios Generales de la empresa, Recicladores, Empresa de Aseo, Gestora de Residuos Peligrosos y la Corporación Autónoma Regional.

\section{Plan de Gestión Integral para el Manejo Integral de residuos sólidos}

El Plan contempla paso a paso las medidas adecuadas de manejo a implementar desde la separación hasta la disposición final, adicionalmente se presentan los programas de formación y educación, Salud Ocupacional y Plan de Contingencia ante situaciones de riesgo en el manejo de residuos.

\section{Plan de Seguimiento}

El Plan de Seguimiento contempla, un flujo grama del procedimiento, actividades de supervisión periódica, auditoria de residuos, control operacional e indicador de gestión.

\section{DISCUSIÓN Y CONCLUSIONES}

A través del estudio y análisis realizado sobre el manejo de residuos sólidos en la empresa Cyrgo SAS, se ha llegado a las siguientes conclusiones:

- En la empresa Cyrgo SAS, aún no se habían implementado acciones para el manejo de residuos sólidos, debido a la falta de cultura, donde cada persona se desarticula de la naturaleza, produciendo una problemática ambiental donde los principales actores son todos los colaboradores de la empresa, que no solo generan impactos negativos dentro de las instalaciones sino que también en el transporte y disposición final. Pero si se corrigen comportamientos negativos a través de campañas culturales de concientización como es la capacitación y mensajes visuales, se podrán generar acciones puntuales y positivas que aportan al medio ambiente y a la salud de las personas, de tal forma que ayuden a que futuras generaciones disfruten de un medio ambiente saludable. 
- El principal problema en la empresa Cyrgo SAS en cuanto al manejo de residuos sólidos, es que no existe un documento técnico el cual contenga, las medidas a implementar para una adecuada gestión de residuos sólidos. Con la implementación y socialización de este documento se tendrá la línea base para que de una manera ordenada y coordinada se inicie con el proceso para la implementación del Plan.

- La escasez de recipientes para segregación de residuos sólidos, en el área administrativa, hace que el personal no tome la cultura de separar desde su origen los residuos generados.

- Existe en la empresa una considerable producción de residuos reciclables que están siendo aprovechados por recicladores, sin embargo podrían gestionarse de manera diferente con empresas recicladoras en el departamento de Nariño con el fin de obtener beneficios económicos para la empresa que pueden invertirse en las mismas actividades de gestión ambiental de la empresa CYRGO SAS.

- Un $100 \%$ de la población encuestada de la empresa Cyrgo SAS, desconoció la disposición final de residuos peligrosos, esto refleja la baja capacitación especialmente en este tipo de residuos, pues como se pudo evidenciar en las encuestas tanto en residuos ordinarios e inertes como de reciclables, tenían algunas bases.

- $\quad$ Cyrgo SAS, como empresa generadora de residuos ha implementado algunas prácticas segregando los residuos peligrosos como pilas, luminarias, RAES, aceite usado y guantes contaminados con epp, sin embargo no cuenta con un sitio de almacenamiento temporal como lo pide la norma, esto puede generar una problemática ambiental y social en las instalaciones pues por sus características estos deben ser almacenados bajo ciertas condiciones.

- $\quad$ No existe un control ni seguimiento a la Empresa Cyrgo SAS en el manejo de residuos peligrosos por parte de la autoridad ambiental de la Región CORPONARIÑO. 


\section{REFERENCIAS}

(1) ALCALDÍA de Envigado. (s.f.). Guía para el adecuado manejo de los residuos sólidos y peligrosos. Alcaldía de Envigado. Secretaria del Medio Ambiente y desarrollo rural.

(2) ALCALDÍA Mayor de Bogotá. (2011). Documento Técnico de Soporte Modificación al Plan de Ordenamiento Territorial de Bogotá. Equidad, Productividad y Sostenibilidad. [En Línea]. Secretaria de Planeación. Bogotá, D. C. Disponible en URL: http://www.sdp.gov.co/portal/page/portal/PortalSDP/POT/QueEs/ DTS.pdf

(3) ANÓNIMO. (s.f.). Contaminación ambiental causada por los residuos sólidos. [En Línea]. Ficad. Disponible en URL:http://www.ficad.org/lecturas/adicional_dos_septima_unidad_eca.pdf

(4) ART-REDES. PNUD. Estrategia de Cooperación Internacional: "Pasto Abierto al Mundo". Municipio de Pasto.

(5) Betancur V, Mariluz; GÓMEZ, Beatriz Elena; BUSTAMANTE M, Ana María. (2011). Guía Para la Gestión de Residuos Peligrosos. Primera Edición. Medellín. Colombia.

(6) CYRGO. (s.f.). Vigas laminadas Wf A 572 Gr 50/ Gerdau. [En Línea]. Ferretería Cyrgo. Disponible en URL: http://www.cyrgo.com.co/index.htm

(7) DELGADO Guerrero, Raúl. (2007). Plan de gestión integral de residuos sólidos PGIRS 2007-2022. [En Línea]. Alcaldía de Pasto. San Juan de Pasto. Disponible en URL: http://www.pasto.gov.co/index.php/ component/phocadownload/category/135-planes-ambientales?download=103:plan-de-gestinintegral-de-residuos-slidos-2007-2022

(8) ELIASSEN, R. (1969). Solid Waste Management: A Comprehensive Assessment of Solid Waste Problems, Practices, and Needs, Office of Science and Technology, Executive Office of the President, Washington, D.C.

(9) FERRER, Jaramillo. MEDINA, Floralba. GÓMEZ, Paola Alexandra. (2010). Plan de gestión integral de residuos sólidos para la vicepresidencia de transporte de Ecopetrol S.A. [En Línea]. Universidad Industrial de Santander. Bucaramanga. Disponible en URL: http://repositorio.uis.edu.co/jspui/ bitstream/123456789/7478/2/133328.pdf

(10) GAIBOR Vaca, NORMA, Yolanda. (2013). Propuesta para el manejo integral de residuos sólidos generados en el Mall de los andes - Ambato. [En línea]. Escuela Superior Politécnica de Chimborazo. Tesis de grado previo a la obtención del título de Ingeniero en Biotecnología Ambiental. Riobamba - Ecuador. Disponible en URL: http://dspace.espoch.edu.ec/bitstream/123456789/2635/1/236T0067.pdf

(11) InSTITUTO COLOMBIANO DE BIENESTAR FAMILIAR (ICBF). (2013). Programa de manejo de residuos sólidos regional Nariño. [En línea]. Instituto Colombiano de Bienestar Familiar -ICBF Regional Nariño. San Juan de Pasto. URL: http://www.icbf.gov.co/portal/page/portal/IntranetICBF/macro_procesos/MP_ ароуо/G_soporte/G_administrativa/PlanesModelos/PP53-MPA1-P5-Programa-de-Manejo-de-ResiduosS\%C3\%B3lidos-Nari\%C3\%B1o-v1.pdf

(12) JONES, B.B. and F. Owen: "Some Notes on the Scientific Aspects of Controlled Tipping," Henry Blacklock and Co., ltd., Printers, Manchester, England, 1934. 
(13) LÓPEZ M, Geovaneli. (2014). Plan de manejo integral de residuos sólidos para hipermercados. [En línea]. Tesis para obtener el título de Ingeniero Industrial. Universidad Nacional Autónoma de México. México. Disponible en URL: http://www.ptolomeo.unam.mx:8080/xmlui/bitstream/ handle/132.248.52.100/4226/Tesis-Geovaneli\%20L\%C3\%B3pez\%20Morfin.pdf?sequence=1

(14) LÓPEZ Rivera, Natalia. (2009). Propuesta de un programa para el manejo de los residuos sólidos en la plaza de mercado de cerete - córdoba. [En Línea]. Trabajo para optar para el título de magistra en gestión ambiental. Universidad Pontificia Javeriana. Bogotá. Disponible en URL: http://repository.javeriana.edu. co/bitstream/10554/6132/1/tesis64.pdf

(15) POT. 2012 - 2015. Cuaderno Diagnostico Sociocultural. [En Línea]. Plan de ordenamiento territorial. Pasto, territorio con sentido. Alcaldía de Pasto. Disponible en URL: http://www.pot.pasto.gov.co/index. php/cuadernos-diagnosticos

(16) SALAZAR falla, María M. (2010). Formulación del plan de manejo integral de residuos sólidos del centro comercial san pedro plaza de la ciudad de Neiva- Huila. [En Línea]. Pontificia Universidad Javeriana. Facultad de estudios ambientales y rurales. Bogotá, DC. Disponible en URL: http://repository.javeriana. edu.co/bitstream/10554/9863/1/tesis78.pdf

(17) SECRETARIA del medio Ambiente y Desarrollo Rural. Guía para el adecuado manejo de residuos sólidos y peligrosos. Secretaria del medio Ambiente y Desarrollo Rural.

(18) SEPÚLVEDA, Luis. (2006). Aprovechamiento de residuos reciclables en Colombia y en el valle de Aburrá. Acodal Occidente. Cali.

(19) WIKIPEDIA. La enciclopedia libre. (2015). San Juan de Pasto. (Modificada por última vez el 15 may 2015 a las 00:10.) [En Línea]. Disponible en URL: http://es.wikipedia.org/wiki/San_Juan_de_Pasto

(20) CANTANHEDE, Álvaro. LA GESTIÓN Y TRATAMIENTO DE LOS RESIDUOS GENERADOS EN LOS CENTROS DE ATENCIÓN DE SALUD. Disponible en URL: http://www.bvsde.ops-oms.org/foro_hispano/BVS/ bvsars/e/fulltext/centros/centros.pdf

(21) DE CONTO, S. M. (2005). Gerenciamento de resíduos sólidos em meios de hospedagem. Análises regionais e globais do turismo brasileiro. São Paulo: Roca, 817-826.

(22) ZARATE QUIÑONES, Rosa - LIZANA VELITA, Sonia, Conocimiento en Conservación Ambiental y Manejo de Residuos Sólidos en la Educación Secundaria y Superior Universitaria en el Distrito de El TamboHuancayo. Disponible en URL: http://es.slideshare.net/mia_18/articulo-cientifico-conservacion-ymanejo-de-residuos-slidos-en-la-educacin-secundaria-y-universitaria-2009

(23) SCHAMBER, Pablo Javier. Revista de la Red de Expertos Iberoamericanos en Gestión de Residuos. (2010). Disponible en URL: file:///C:/Users/SONY/Downloads/N4_Revista_Digital_de_la_REI_en_GESTION_DE_ RESIDUOS.pdf

(24) AGUDELO, Ruth Marina - RENDÓN, Iván Darío - PALACIO, Jorge Andrés. Gestión integral de residuos sólidos peligrosos y cumplimiento de normas de bioseguridad en laboratorios de tanatopraxia, Medellín, 2001. Disponible en URL: http://www.redalyc.org/pdf/120/12021104.pdf

(25) PLAZA, Gloria - Zapata, Omar. Residuos y Salud. Disponible en URL: http://www.scielo.org.ar/scielo. php?script=sci_arttext\&pid=S1851-75872011000200005.

(26) CASTRILLÓN QUINTANA, Olivia - PUERTA ECHEVERRI, Silvia María. Impacto del manejo integral de los residuos sólidos en la Corporación Universitaria Lasallist. Disponible en URL: http://hdl.handle. net/10567/174 